\title{
Outcomes at 7 years for babies who developed neonatal necrotising enterocolitis: the ORACLE Children Study
}

\author{
Katie Pike, ${ }^{1}$ Peter Brocklehurst, ${ }^{2}$ David Jones, ${ }^{3}$ Sarah Kenyon, ${ }^{4}$ Alison Salt, ${ }^{5}$ \\ David Taylor, ${ }^{6}$ Neil Marlow²
}

- Supplementary appendix A is available online only. To access this file please visit the journal online (http://adc.bmj. com/content/early/recent)

${ }^{1}$ Bristol Heart Institute, University of Bristol, Bristol, UK

2Institute for Women's Health, University College London, London, UK

${ }^{3}$ Health Sciences Department, University of Leicester, Leicester, UK

${ }^{4}$ School of Health and Population Sciences, University of Birmingham, Birmingham, UK ${ }^{5}$ Great Ormond Hospital for Sick Children and Institute of Child Health, University College London, London, UK

${ }^{6}$ Maternal and Newborn Health Initiative, International Federation of Gynaecology and Obstetrics, London, UK

\section{Correspondence to}

Neil Marlow, Institute for Women's Health, UCL Institute for Women's Health, 74 Huntley Street, London WC1E 6AU, UK;

n.marlow@ucl.ac.uk

Received 26 April 2011 Accepted 7 December 2011

Published Online First 20 January 2012

\section{ABSTRACT \\ Background Within the ORACLE Children Study \\ Cohort, the authors have evaluated long-term consequences of the diagnosis of confirmed or suspected neonatal necrotising enterocolitis (NEC) at age of 7 years.}

Methods Outcomes were assessed using a parental questionnaire, including the Health Utilities Index (HUI-3) to assess functional impairment, and specific medical and behavioural outcomes. Educational outcomes for children in England were explored using national standardised tests. Multiple logistic regression was used to explore independent associates of NEC within the cohort. Results The authors obtained data for $119(77 \%)$ of 157 children following proven or suspected NEC and compared their outcomes with those of the remaining 6496 children. NEC was associated with an increase in risk of neonatal death (OR 14.6 (95\% Cl 10.4 to 20.6)). At 7 years, NEC conferred an increased risk of all grades of impairment. Adjusting for confounders, risks persisted for any HUI-3 defined functional impairment ladjusted OR $1.55(1.05,2.29))$, particularly mild impairment (adjusted OR $1.61(1.03,2.53))$ both in all NEC children and in those with proven NEC, which appeared to be independent. No behavioural or educational associations were confirmed. Following NEC, children were more likely to suffer bowel problems than non-NEC children (adjusted OR $3.96(2.06,7.61)$ ).

Conclusions The ORACLE Children Study provided opportunity for the largest evaluation of school age outcome following neonatal NEC and demonstrates significant long-term consequences of both gut function (presence of stoma, admission for bowel problems and continuing medical care for gut-related problems) and motor, sensory and cognitive outcomes as measured using HUI-3.

\section{INTRODUCTION}

Neonatal necrotising enterocolitis (NEC) is a devastating condition occurring primarily in very preterm children in the weeks after birth and is thought to result from a combination of factors, namely hypoxia, infection and feeding. ${ }^{1}$ Because of high intrinsic mortality and morbidity, it is monitored within neonatal and perinatal trials as an adverse outcome. NEC is associated with an increased frequency of neurodevelopmental problems in survivors, mainly evaluated in infancy, ${ }^{23}$ and potentially mediated by white matter injury associated with the primary illness. ${ }^{4}$

\section{What is known about this topic}

- Necrotising enterocolitis is a devastating condition with high mortality and neonatal morbidity.

- It is associated with an increased prevalence of disability in infancy.

- Longer-term sequelae are rarely described.

\section{What this study adds}

- Necrotising enterocolitis (NEC) confers an increased risk of functional impairments in middle childhood which has an impact on family life.

- Necrotising enterocolitis also is associated with continuing bowel dysfunction in middle childhood.

ORACLE evaluated the effects of prescription of erythromycin or co-amoxiclav for women with either preterm rupture of the membranes (PROM) or spontaneous preterm labour (SPL) with intact membranes and no overt infection, using a $2 \times 2$ factorial design. ORACLE collected limited data regarding NEC, namely whether the baby had had the condition and whether that was suspected or confirmed by an x-ray/operation.

Among babies whose mothers had PROM, co-amoxiclav prescription was associated with increased risk of NEC, ${ }^{5}$ the reason for which is unknown. In contrast, for women with PROM, erythromycin was associated with prolongation of pregnancy and improvements in short-term maternal and neonatal morbidity; for singletons, there was a reduction in the composite primary outcome (death or abnormal cerebral ultrasound or use of supplemental oxygen at 36 weeks postmenstrual age). For women with SPL, there was no evidence of either benefit or harm at discharge from hospital. ${ }^{5}$

The ORACLE Children Study (OCS) sought follow-up information for surviving children at 7 years of age in the UK using a parent-report postal questionnaire. ${ }^{6}$ The primary outcome was defined as the presence of any level of functional impairment using the Multi-Attribute Health Status (MAHS) classification system. ${ }^{7}$ Educational attainment 
at 7 years was assessed for children resident in England using results from National Curriculum tests at Key Stage 1.

We found that for children whose mothers had PROM, the prescription of antibiotics seemed to have little effect on the health and educational attainment of children at 7 years. ${ }^{8}$ For children whose mothers had SPL, the prescription of erythromycin (with or without co-amoxiclav) was associated with an increase from $38 \%$ to $42 \%$ in the numbers of children with any level of functional impairment. ${ }^{9}$ There was also about twofold increase in the risk of cerebral palsy (CP) associated with the use of either antibiotic from just under $2 \%$ to just over $3 \%$ and perhaps particularly with the use of both.

We have taken the opportunity of the OCS to evaluate longer-term outcomes for children with NEC than have been previously reported. We hypothesised that this group of children would be at particular risk of functional impairments in middle childhood, leading to poorer educational attainment.

\section{METHODS}

\section{Population}

Among children born between July 1994 and August 2000 to mothers resident in the UK within ORACLE, 209 were identified to have NEC - for 83 children, this was considered proven and for 126 this was suspected with no radiological or surgical confirmation. Of these, 52 died during their original admission and three after discharge from maternity services; we obtained information on the outcome for 119 of 154 eligible children at 7 years of age $(77 \%)$ as part of the OCS. These outcomes were compared with those of 6439 children whose mothers responded from a total of 8729 children and who did not have $\mathrm{NEC}$ in the neonatal period (figure 1).

\section{ORACLE Children Study}

OCS was carried out between 2002 and 2007. Details of the contact and tracing of the cohort are described in the original reports $^{89}$ and appropriate approval to perform the study was granted by the West Midlands Multi-centre Research Ethics Committee. Parental report outcomes included the Health Utilities Index (HUI) from which the MAHS is derived, ${ }^{7}$ the Strengths and Difficulties Questionnaire (SDQ) ${ }^{10}$ together with specific questions on respiratory symptoms, hospital admissions, convulsions, other specific medical conditions and demographic data. We used results from National Curriculum tests (Key Stage 1), done at 7 years of age to assess the children's educational attainment for residents in England for those children whose parents gave permission for us to obtain them from the school/local education authority. Data concerning social disadvantage, educational level and child poverty risk were derived from national data (www.neighborhood.statistics.gov.uk). OCS data were combined with results from the original trial for analysis.

\section{Statistical analysis}

The sample size was prespecified by the original ORACLE trial and the response to the OCS. An indicative power calculation assuming $40 \%$ prevalence of any functional impairment for the children without NEC showed that at $80 \%$ power and

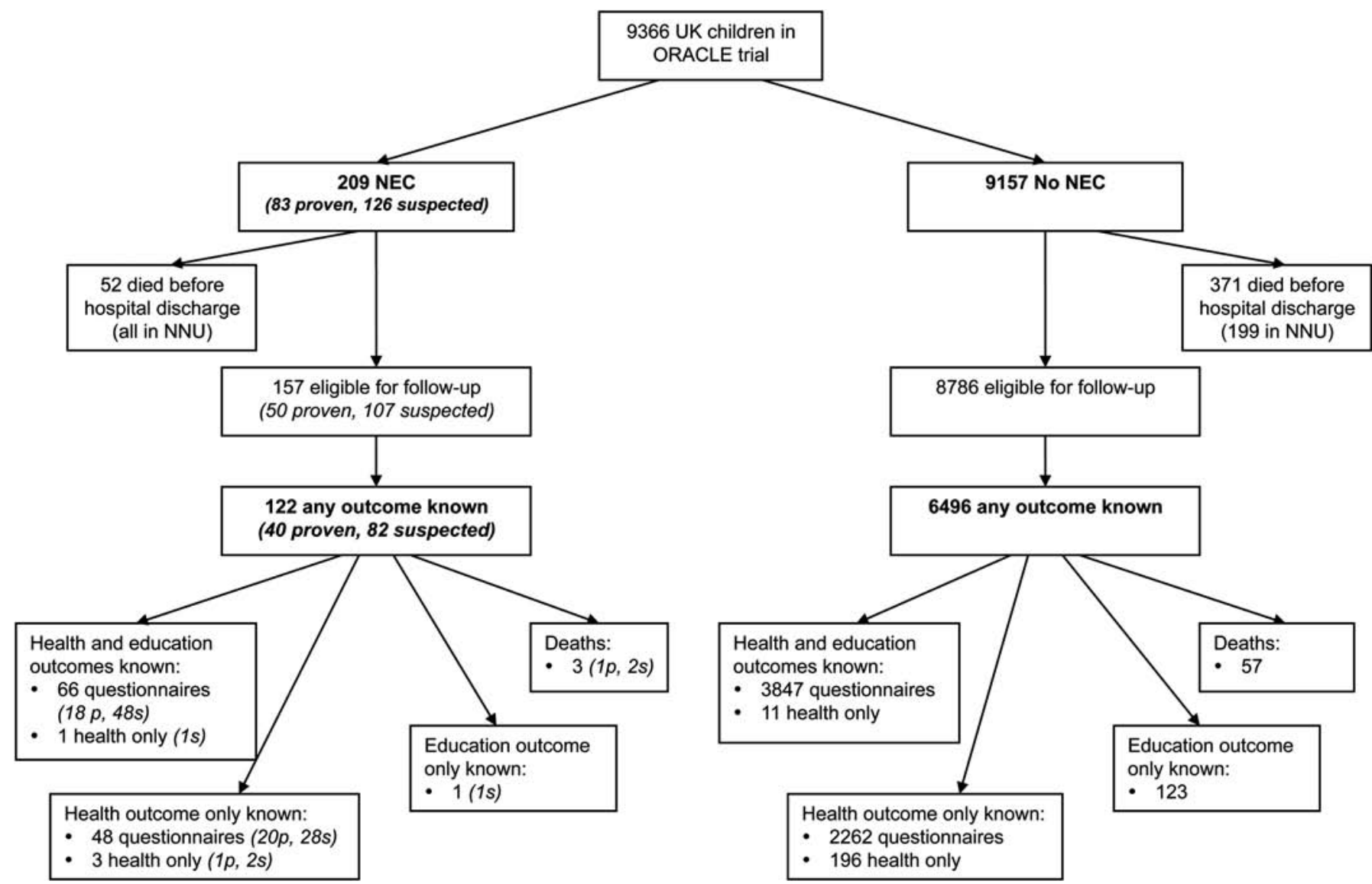

Figure 1 Derivation of the two populations compared in the study from the original ORACLE population. On the necrotising enterocolitis (NEC) side of the flow chart, the abbreviations ' $p$ ' and 's' are used to denote proven and suspected NEC, respectively. 
Table 1 OR associated with NEC for functional impairment (derived from HUI-3 completed by parents) in the ORACLE Children Study

\begin{tabular}{|c|c|c|c|c|c|c|c|c|}
\hline & $\begin{array}{r}\text { NEC } \\
n=114 \\
\end{array}$ & $\begin{array}{l}\text { No NEC } \\
\mathrm{n}=6109\end{array}$ & \multicolumn{2}{|c|}{ Unadjusted model } & \multicolumn{2}{|c|}{ Model 1 (antenatal factors) } & \multicolumn{2}{|c|}{$\begin{array}{l}\text { Model } 2 \text { (antenatal and } \\
\text { neonatal factors) }\end{array}$} \\
\hline Any impairment & $64(56.1 \%)$ & $2417(39.6 \%)$ & 1.87 & (1.30 to 2.71$)$ & 1.74 & (1.18 to 2.55$)$ & 1.55 & (1.05 to 2.28$)$ \\
\hline Moderate impairment* & $21(18.4 \%)$ & $680(11.1 \%)$ & 2.18 & (1.31 to 3.64$)$ & 1.95 & (1.14 to 3.33$)$ & 1.70 & (0.99 to 2.91 ) \\
\hline Severe impairment* & $9(7.9 \%)$ & $362(5.9 \%)$ & 1.76 & (0.86 to 3.59 ) & 1.37 & (0.65 to 2.87$)$ & 1.03 & (0.47 to 2.27$)$ \\
\hline $\begin{array}{l}\text { Three or more abnormal } \\
\text { attributes }\end{array}$ & $18(15.8 \%)$ & $520(8.5 \%)$ & 1.97 & (1.19 to 3.29$)$ & 1.43 & (0.84 to 2.43 ) & 1.13 & (0.65 to 1.96$)$ \\
\hline
\end{tabular}

*Models compare children with the level of impairment to those with no impairment (eg, for mild impairment, those with moderate and severe impairment are excluded). No missing data.

HUI, Health Utilities Index; NEC, necrotising enterocolitis.

$5 \%$ significance level, this study could detect a relative difference between the children without NEC and those with NEC of $34 \%$ (ie, a prevalence of $54 \%$ for children with NEC).

Five sets of outcomes were defined for this analysis: death, functional impairment (presence of any impairment in three categories and impairment for three or more attributes from HUI-3), behaviour (presence of abnormal or borderline scores for emotional symptoms, conduct problems, hyperactivity, peer problems, pro-social behaviour, total difficulties and impact); medical conditions (presence of $\mathrm{CP}$, seizures, attention deficit hyperactivity disorder (ADHD) and bowel problems from parental questionnaire) and educational attainment (achieving below level 2 at Key Stage 1 tests in reading, writing and mathematics).

Initial unadjusted analyses were performed to determine the extent to which the occurrence of NEC predicts the OCS outcome. Multiple logistic regression models were then used to allow adjustment for any factors that may confound the relationship between NEC and the relevant outcome. Two groups of possible confounders were identified: model 1 - characteristics present before birth: maternal age, gestational age at birth, birth weight for gestation ' $Z$ ' score, mode of birth, antenatal steroid use, sex, multiple birth, ethnicity (white/non-white), housing type (owned/non-owned), PROM, erythromycin and co-amoxiclav, postnatal prescription of antibiotics to the mother; model $2-$ characteristics present before birth plus neonatal outcomes: as above, and positive blood culture in the baby, respiratory distress syndrome, use of surfactant, ventilation, supplemental oxygen at 28 days and abnormal cerebral ultrasound.

A significance level of $10 \%$ was used for selection of variables at all stages of model building. Categorical variables were fitted with the most common category as the reference category. Highly correlated variables (eg, ventilation and oxygenation at 28 days) were not simultaneously included in any models. Results from both adjusted and unadjusted analyses are displayed as adjusted OR and $95 \%$ CI. Full details of the factors adjusted for in all of the models are given in online supplementary Appendix A.

There were no missing data for any of the potential confounding factors. Of the children whose mothers responded to the OCS questionnaires, there is very little missing data for OCS outcomes. The amount of missing data is described for each outcome. No formal adjustment was made for multiple testing. However, when interpreting results, consideration was made for the plausibility and direction of effect estimates. All analyses were performed in Stata version 10.

\section{RESULTS}

Non-responders to the OCS had on average younger mothers, experienced less neonatal morbidity and were from more deprived areas than non-responders. In addition, more nonresponding children scored below level 2 in Key Stage 1 tests and were non-white compared with the responders. $\left({ }^{56}\right)$.

\section{Death before discharge from neonatal admission}

Fifty-two of 209 (25\%) children with NEC died before discharge compared with 199/8985 (2.2\%) children without NEC (OR (95\% CI): 14.6 (10.4 to 20.6)). OR were reduced in the adjusted analyses (model 1: 2.30 (1.54 to 3.44); model 2: 2.08 (1.40 to 3.10)); NEC is thus an independent risk factor for hospital deaths. Gestation at birth, caesarean section or breech birth (compared with spontaneous vaginal birth), ventilation and abnormal cerebral ultrasound were all found to be associated with death predischarge and were included in adjusted models.

\section{Functional impairment}

Using the parent report HUI-3 classification of disability, $56 \%$ (64 of 114) children with NEC had any functional impairment compared with $40 \%$ (2417 of 6109) without NEC; NEC was associated with an increased risk of any impairment (1.87 (1.30, 2.71)) and significant increased risk at each level of severity, with the exception of severe impairment (table 1). Adjusted analyses suggested statistically significant differences for any functional impairment (OR $1.55(1.05,2.28)$ ) and mild impairment (OR 1.61 $(1.03,2.53)$, and a marginal difference for moderate impairment (OR $1.70(0.99,2.91)$ (table 1); however, event rates for both moderate and severe impairments are relatively low. The association between NEC and three or more abnormal attributes was no longer evident after adjustment for other neonatal factors.

Factors that were commonly included in adjusted models were maternal age, gestation at birth, ventilation and abnormal cerebral ultrasound.

\section{Behaviour}

In unadjusted analyses for abnormal or borderline results from the SDQ, all point estimates of OR comparing children with NEC to those without NEC were greater than one but significant associations were only seen for the impact score (OR 1.66 (1.11, 2.50); table 2). Once adjustment for all other factors was performed, there was no clear evidence of statistically significant relationships between NEC and any behavioural outcomes. Factors consistently included in the models were maternal age, markers of gestation (gestation at birth or at PROM), multiple births and ventilation/oxygenation at 28 days age. For full details, see online supplementary Appendix A.

\section{Educational outcomes}

In unadjusted analyses, poor educational attainment in each Key Stage 1 subject was strongly associated with neonatal NEC 
Table 2 OR associated with NEC for abnormal or borderline scores on parent completed Strengths and Difficulties Questionnaires

\begin{tabular}{|c|c|c|c|c|c|c|c|c|}
\hline & $\begin{array}{c}\text { NEC } \\
\mathrm{n}=114\end{array}$ & $\begin{array}{l}\text { No NEC } \\
n=6109\end{array}$ & \multicolumn{2}{|c|}{ Unadjusted model } & \multicolumn{2}{|c|}{ Model 1 (antenatal factors) } & \multicolumn{2}{|c|}{$\begin{array}{l}\text { Model } 2 \text { (antenatal and } \\
\text { neonatal factors) }\end{array}$} \\
\hline Emotional symptoms & $26(22.8 \%)$ & $1210(19.9 \%)$ & 1.17 & (0.75 to 1.82$)$ & 1.31 & (0.84 to 2.04$)$ & 1.04 & (0.66 to 1.65$)$ \\
\hline Hyperactivity & $39(34.2 \%)$ & $1615(26.6 \%)$ & 1.41 & (0.96 to 2.08$)$ & 1.30 & (0.87 to 1.95$)$ & 1.09 & (0.72 to 1.65$)$ \\
\hline Peer problems & $32(28.1 \%)$ & $1441(23.7 \%)$ & 1.23 & (0.82 to 1.86$)$ & 1.37 & (0.90 to 2.09$)$ & 1.17 & (0.76 to 1.80$)$ \\
\hline Impact score & 33 (29.7\%) & 1178 (19.7\%) & 1.66 & (1.11 to 2.50 ) & 1.78 & (1.17 to 2.70$)$ & 1.32 & (0.85 to 2.05 ) \\
\hline
\end{tabular}

There are some missing data: emotional symptoms, $36(0.6 \%)$; conduct problems, $35(0.6 \%)$; hyperactivity, 39 (0.6\%); peer problems, 33 (0.5\%); pro-social behaviour, 24 $(0.4 \%)$; total difficulties, $42(0.7 \%)$; impact, $138(2.2 \%)$.

NEC, necrotising enterocolitis.

Table 3 OR associated with NEC for educational attainment below level 2 at Key Stage 1 (parental permission required for residents in England)

\begin{tabular}{|c|c|c|c|c|c|c|c|c|}
\hline & $\frac{\text { NEC }}{n=68}$ & $\frac{\text { No NEC }}{n=3981}$ & \multicolumn{2}{|c|}{ Unadjusted model } & \multicolumn{2}{|c|}{ Model 1 (antenatal factors) } & \multicolumn{2}{|c|}{$\begin{array}{l}\text { Model } 2 \text { (antenatal and } \\
\text { neonatal factors) }\end{array}$} \\
\hline Reading & $21(30.9 \%)$ & $653(16.5 \%)$ & 2.18 & (1.30 to 3.65 ) & 1.97 & (1.14 to 3.38$)$ & 1.68 & (0.97 to 2.91$)$ \\
\hline Maths & $12(17.6 \%)$ & $393(9.9 \%)$ & 1.89 & (1.01 to 3.54$)$ & 1.36 & (0.71 to 2.62 ) & 1.15 & (0.59 to 2.25$)$ \\
\hline
\end{tabular}

There is a small amount of missing data: reading, $15(0.4 \%)$; writing, $6(0.1 \%)$; maths, $7(0.2 \%)$.

NEC, necrotising enterocolitis.

(table 3). However, after adjustment for other factors, there was no formal evidence of statistically significant relationships between education and NEC, although this is marginal for reading (OR $1.68(0.97,2.91))$. In addition, all estimated ORs are greater than one.

\section{Medical conditions}

On univariate analysis, associations were found between NEC and CP (8/118 (7\%) vs 159/6316 (3\%); OR $2.77(1.33,5.77))$, and parent report of ADHD (18/118 (15\%) vs 462/6316 (7\%); OR 2.24 (1.34, 3.72); table 4). After adjustment, there was no formal statistical evidence of increased CP or increased ADHD associated with NEC. However, the event rates are low.

Significant associations were found between NEC and later bowel problems in childhood (18/118 (7\%) vs 202/6316 $(3 \%)$; OR $5.34(3.17,8.98))$ and particularly the presence of a stoma (OR $4.16(1.78,9.73)$ ), as might be anticipated, but also of 'other bowel problems' (OR 5.59 (3.00, 10.4)). These were further defined in free text and comprised hospital admissions for constipation, diarrhoea, stomach troubles or being under care of a doctor for bowel problems). Adjustment for other factors suggested strong evidence of NEC being an independent risk factor for all bowel problems and non-stoma bowel problems (method 2 ORs $3.61(2.08,6.25)$ and $3.96(2.06,7.61)$, respectively). Evidence was weaker for bowel stoma (method 2 OR 2.35 (0.96, 5.74)). Again, event rates are relatively low. Important factors influencing the relationship between NEC and bowel problems at age seven included gestation at birth, multiple births, antenatal steroid use and postnatal surfactant use/respiratory distress syndrome.

\section{DISCUSSION}

NEC remains a devastating condition that arises during neonatal intensive care primarily of the very preterm infant. It is associated with a high frequency of shock and coagulopathy and will frequently require laparotomy and stoma formation.
Postoperatively, there is a risk of short bowel syndrome with its attendant complications. In this study, we have confirmed the high neonatal mortality but in addition shown that NEC, whether suspected or proven, independently confers an excess of both functional impairments and childhood bowel problems. The small number of children with proven NEC poses a problem of small sample size but analysing as three groups (proven NEC, suspected NEC and no NEC) does not alter these conclusions. Reassuringly, there do not appear to be strong associations with behavioural or educational problems. This study provides useful information for clinicians and parents as to the long-term consequences of this condition and confirms the findings from other outcome studies of groups comprising severe neonatal disease. ${ }^{23}$ This also confirms our anxiety concerning the use of co-amoxiclav to treat women who present with PROM as its use was associated with an increased prevalence of NEC in the ORACLE I trial. ${ }^{5}$

We chose to structure our analyses using a sequential pattern of analysis to obtain as much information about the nature of the associations observed. In model 1, we identified pre-existing factors that were not modifiable by alternative perinatal management strategies. In this group, we placed the trial interventions because of the finding of the main ORACLE trial. In the second group (model 2), we placed factors we had recorded which would have placed the infant at an increased risk of NEC. No data were collected regarding the timing of onset of milk feeds or of the use of formula milk; neither was the use of inotropic support nor antenatal measures of fetal health, in particular Doppler studies of fetal flow velocities, so we simply added two markers of sepsis risk - treatment of the mother postnatally for infection and the presence of a positive blood culture in the child. In this group, we added items that were coexistent with NEC, as they may have mediated the effect of NEC on the outcome concerned. This produces interesting results, despite the risk of over-adjustment. In terms of functional impairments, there is a strong independent relationship of NEC with any impairment and with mild impairment. The 
Table 4 OR associated with NEC for neurological or bowel problems reported by parents in the ORACLE Children Study

\begin{tabular}{|c|c|c|c|c|c|c|c|c|}
\hline & $\begin{array}{r}\text { NEC } \\
n=118\end{array}$ & $\frac{\text { No NEC }}{n=6316}$ & \multicolumn{2}{|c|}{ Unadjusted model } & \multicolumn{2}{|c|}{ Model 1 (antenatal factors) } & \multicolumn{2}{|c|}{$\begin{array}{l}\text { Model } 2 \text { (antenatal and } \\
\text { neonatal factors) }\end{array}$} \\
\hline Cerebral palsy & $8(6.8 \%)$ & $159(2.5 \%)$ & 2.77 & $(1.33,5.77)$ & 1.23 & (0.58 to 2.63 ) & 0.87 & (0.39 to 1.95$)$ \\
\hline ADHD & $18(15.3 \%)$ & $462(7.3 \%)$ & 2.24 & $(1.34,3.72)$ & 2.08 & (1.22 to 3.54$)$ & 1.60 & (0.92 to 2.78 ) \\
\hline Any bowel problem & $18(15.3 \%)$ & $202(3.2 \%)$ & 5.34 & $(3.17,8.98)$ & 3.69 & (2.12 to 6.43 ) & 3.61 & (2.08 to 6.25$)$ \\
\hline
\end{tabular}

No missing data.

ADHD, attention deficit hyperactivity disorder; NEC, necrotising enterocolitis.

effect of the association with moderate impairment is no longer evident when other co-morbidities and mediating variables are added in. However, the number of children with moderate impairments is low. NEC is itself associated with an increased need for ventilation, an increased risk of bronchopulmonary dysplasia and of intraventricular haemorrhage. Hence, some of the effect of NEC on functional impairment may have been mediated through these excess risks. Of interest is that selecting out only those children with proven NEC demonstrates a persisting association with moderate functional impairment. Similarly, there is some face validity to these associations provided by the parent report of seizure disorders and of ADHD diagnoses as more prevalent within children who had NEC.

The size of the population in the OCS meant that assessment of the children by parental questionnaire was the most practical and cost-effective way of obtaining information. We included validated tools within the questionnaire where possible. More subtle treatment differences may thus have been missed. There is also the potential for health conditions to be either overreported or under-reported. We acknowledge that the response rate obtained, particularly for the children's educational attainment, may allow some bias, but there was no evidence of differential non-response bias between the randomised groups.

While mortality in extremely and very low birthweight babies has decreased significantly over the past 20 years, mortality from NEC has significantly increased in England and Wales. ${ }^{11}$ Few studies have evaluated long-term functional or neurodevelopmental outcome after neonatal NEC specifically and the median follow-up found by a recent systematic review was found to be 20 months (range $12-156) ;^{23}$ so, this study offers evidence about longer-term development at 7 years in a large group of children. This study is unique in that it was able to look at behaviour, other medical conditions and educational attainment as well as neurodevelopment, which has been the focus of other studies. NEC is associated with a florid inflammatory response, which may play a role in the poor growth and neurodevelopment found in survivors; increased serum levels of pro-inflammatory cytokines have been associated with abnormal cognitive and psychomotor outcomes. ${ }^{12}$

The association with stoma formation and other bowel problems is interesting. Following NEC, stomas are usually temporary unless there is significant loss of bowel and short bowel syndrome. However, even then closure is usually attempted suggesting ongoing bowel problems beyond the first year. Even where there is no stoma, we have demonstrated an increased risk of ongoing general bowel problems following NEC, which is rarely recognised in the literature, although there may be some over-reporting of symptoms, as parents were aware that their child had NEC.
In this study, we have demonstrated ongoing morbidity at 7 years associated with NEC occurring in the neonatal period among the children of women recruited to ORACLE. This extends outside specific bowel symptoms to functional impairments, ongoing seizure disorders and to a diagnosis of ADHD reported by parents. Some of this morbidity is mediated through the well-described pathways of major neonatal morbidities themselves associated with NEC.

Acknowledgements The authors thank all the women and children who took part in this study and the obstetricians and midwives who recruited them to the original ORACLE trials

Funding Medical Research Council Both the ORACLE Trial and the Children Study were funded by the UK Medical Research Council (MRC) ISRCT Number 52995660 and sponsored by University Hospitals of Leicester and approved by their research and development directorate. NM receives part funding from the Department of Health's NIHR Biomedical Research Centres funding scheme at UCLH/UCL.

Competing interests None.

Ethics approval West Midlands Multi-centre Research Ethics Committee.

Provenance and peer review Not commissioned; externally peer reviewed.

\section{REFERENCES}

1. Neu J, Walker WA. Necrotizing enterocolitis. N Eng/ J Med 2011;364:255-64.

2. Rees CM, Pierro A, Eaton S. Neurodevelopmental outcomes of neonates with medically and surgically treated necrotizing enterocolitis. Arch Dis Child Fetal Neonatal Ed 2007;92:F193-8.

3. Schulzke SM, Deshpande GC, Patole SK. Neurodevelopmental outcomes of very low-birth-weight infants with necrotizing enterocolitis: a systematic review of observational studies. Arch Pediatr Adolesc Med 2007;161:583-90.

4. Shah DK, Doyle LW, Anderson PJ, et al. Adverse neurodevelopment in preterm infants with postnatal sepsis or necrotizing enterocolitis is mediated by white matter abnormalities on magnetic resonance imaging at term. J Pediatr 2008;153:170-5, 175.e1.

5. Kenyon SL, Taylor DJ, Tarnow-Mordi W. Broad-spectrum antibiotics for preterm, prelabour rupture of fetal membranes: the ORACLE I randomised trial. ORACLE Collaborative Group. Lancet 2001;357:979-88

6. Kenyon S, Brocklehurst P, Jones D, et al. MRC ORACLE Children Study. Long term outcomes following prescription of antibiotics to pregnant women with either spontaneous preterm labour or preterm rupture of the membranes. BMC Pregnancy Childbirth 2008;8:14.

7. Saigal S, Feeny D, Furlong W, et al. Comparison of the health-related quality of life of extremely low birth weight children and a reference group of children at age eight years. J Pediatr 1994;125:418-25.

8. Kenyon S, Pike K, Jones DR, et al. Childhood outcomes after prescription of antibiotics to pregnant women with preterm rupture of the membranes: 7-year follow-up of the ORACLE I trial. Lancet 2008;372:1310-18.

9. Kenyon S, Pike K, Jones DR, et al. Childhood outcomes after prescription of antibiotics to pregnant women with spontaneous preterm labour: 7-year follow-up of the ORACLE II trial. Lancet 2008;372:1319-27.

10. Goodman R. The Strengths and Difficulties Questionnaire: a research note. J Child Psychol Psychiatry 1997;38:581-6.

11. Rees CM, Eaton S, Pierro A. Treatment of necrotizing enterocolitis. N Eng/ J Med 2006;355:847; author reply 847.

12. Lodha A, Asztalos E, Moore AM. Cytokine levels in neonatal necrotizing enterocolitis and long-term growth and neurodevelopment. Acta Paediatr 2010;99:338-43 


\section{Corrections}

Pike K, Brocklehurst P, Jones D, et al. Outcomes at 7 years for babies who developed neonatal necrotising enterocolitis: the ORACLE Children Study (Arch Dis Child Fetal Neonatal Ed 2012;97:F318-22). This paper was published with an incorrect doi. It should be doi:10.1136/archdischild-2011-300244.

Arch Dis Child Fetal Neonatal Ed 2013;98:e1. doi:10.1136/archdischild-2011-300244 